\title{
Hipertensión pulmonar como manifestación de la telangiectasia hemorrágica hereditaria o síndrome de Osler-Weber-Rendu
}

\author{
Pulmonary hypertension as manifestation of hereditary \\ hemorrhagic telangiectasia, or Osler-Weber-Rendu \\ syndrome
}

Ángela Maria Giraldo M., MD. ${ }^{(1)}$; Rafael Conde Camacho, MD. (2); Fabio Andrés Varón V., MD. ${ }^{(3)}$

\section{Resumen}

La telangiectasia hemorrágica hereditaria o síndrome de Osler-Weber-Rendu, es una enfermedad infrecuente, cuya prevalencia estimada es de entre 1:5.000 y 1:8.000 personas. Existen tasas más elevadas en ciertas áreas geográficas como Curazao y Bonaire, pero no es posible definir con exactitud las estadísticas debido al desconocimiento de la enfermedad en la mayoría de los pacientes que la padecen. Es un trastorno autosómico dominante con penetrancia variable que se caracteriza por epistaxis, telangiectasias y malformaciones vasculares en distintos territorios, tales como pulmonar, gastrointestinal, hepático y cerebral.

Palabras clave: síndrome de Osler-Weber-Rendu, hipertensión pulmonar, malformaciones vasculares, activina tipo receptor kinasa 1.

\begin{abstract}
Hereditary hemorrhagic telangiectasia, or Osler-Weber-Rendu syndrome, is an uncommon disease whose estimated prevalence ranges from 1:5.000 to 1:8.000. There are higher rates in certain geographic areas, such as Curazao and Bonaire, but it is impossible to accurately define the statistics, due to lack of knowledge of the existence of the disease in most sufferers. It is a dominant autosomal disorder with variable penetrance, characterized by epistaxis, telangiectasia, and vascular malformations in many territories, such as the pulmonary, gastrointestinal, hepatic, and cerebral vascular beds (1).
\end{abstract}

Keywords: Osler-Weber-Rendu syndrome, pulmonary hypertension, vascular malformations, activin type-1 kinase receptor.

\section{Introducción}

Esta enfermedad fue reconocida por primera vez en el siglo XIX debido a la presencia de hemorragias nasales y del tracto gastrointestinal. Posteriormente fue mejor caracterizada por Rendu, Osler, Weber y Hanes como una entidad con carácter hereditario, autosómica dominante. Para esta época las manifestaciones clínicas identificadas eran epistaxis, sangrado gastrointestinal, anemia por déficit de hierro y telangiectasias mucocutáneas. Sólo hasta 1940, con el desarrollo de las imágenes diagnósticas, se estableció la asociación de esta entidad con la presencia de malformaciones arteriovenosas en el pulmón, el hígado y la circulación cerebral. Se han determinado dos tipos de lesiones vasculares asociadas con esta patología: telangiectasias (dilatación de vasos sanguíneos pequeños: arteriolas,
(1) Fellow de Neumología, Universidad de la Sabana. Fundación Neumológica Colombiana, Santa Fe de Bogotá.

${ }^{(2)}$ Neumólogo, Fundación Neumológica Colombiana, Santa Fe de Bogotá.

${ }^{(3)}$ Neumólogo Intensivista, Fundación Neumológica Colombiana, Santa Fe de Bogotá. Correspondencia: Angela María Giraldo. Correo electrónico: angelagiral@gmail.com Recibido: 01/05/14. Aceptado: 10/06/14. 
vénulas o capilares ubicados principalmente en piel y mucosas) y malformaciones arteriovenosas (comunicación anormal entre arterias y venas en parénquima pulmonar, hepático y cerebral). Hoy se sabe que la mitad de los pacientes tienen compromiso de la vasculatura pulmonar.

\section{Caso}

Paciente de género masculino, de 40 años edad, con cuadro de dos años de disnea de esfuerzo progresiva; en el momento MRC 4/4, dolor torácico y edema periférico. Durante el examen físico se documentaron signos asociados con hipertensión pulmonar y falla del ventrículo derecho: reforzamiento del segundo ruido cardíaco, soplo holosistólico que aumentaba con la inspiración, hepatomegalia, hígado pulsátil, edema periférico de miembros inferiores, ascitis y lesiones tipo dilataciones vasculares eritemato-violáceas en cavidad oral y lengua (telangiectasias orales) (figura 1). Describió episodios de epistaxis espontánea recurrente en la adolescencia y síndrome anémico crónico. Antecedente familiar de epistaxis en hermanas.

Con estos hallazgos se hizo impresión diagnóstica de hipertensión pulmonar y se sospechó telangiectasia hemorrágica hereditaria $(\mathrm{THH})$. Se solicitaron varios paraclínicos entre ellos: radiografía de tórax en la que se observó dilatación de los segmentos principales de las arterias pulmonares (figura 2); tomografía de tórax en la que se identificaron varias imágenes de aspecto nodular lobuladas de contorno
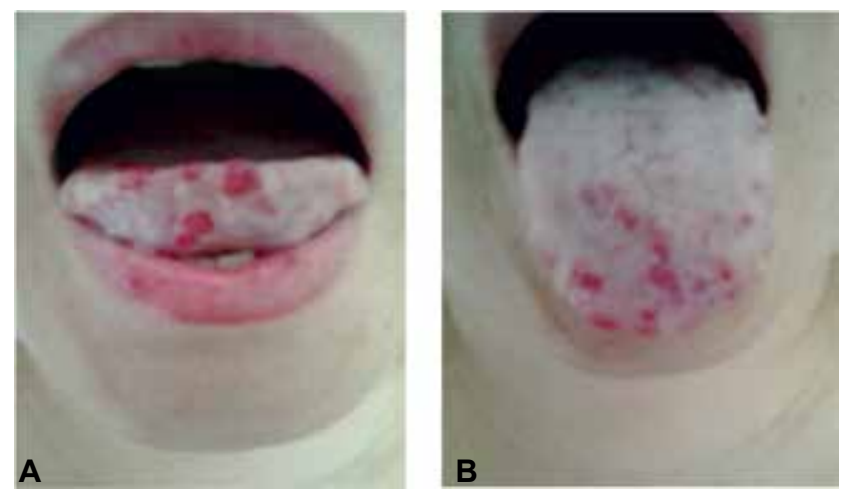

Figura 1. Panel A y B. Dilataciones de pequeños vasos sanguíneos en dorso de lengua y mucosa oral (telangiectasias orales) (imágenes del paciente). bien definido y densidad de tejido blando localizadas en el segmento superior del lóbulo inferior izquierdo, en el segmento anterior del lóbulo superior derecho y en la língula con diámetros entre $3 \mathrm{~mm}$ y $5 \mathrm{~mm}$, así como de una imagen hiperdensa de 2 mm localizada en el segmento IV hepático asociada a signos de hipertensión pulmonar precapilar severa (figura 3).

Dados los hallazgos tomográficos se decidió realizar una gammagrafía pulmonar con tecnecio $99 \mathrm{mTc}$ y macroagregados de albúmina para valorar la presencia de cortocicuito y vasodilatación intrapulmonar, la cual confirmó una distribución irregular del trazador en el parénquima pulmonar con áreas alternas de híper e hipocaptación y depósito del material radioactivo en el cerebro mayor al 7\%, así como en los riñones, que indicaba la presencia de cortocircuito de derecha a izquierda (figura 4).

Como parte del estudio de THH con compromiso pulmonar secundario a malformaciones arteriovenosas y con sospecha de compromiso del parénquima hepático, se realizó resonancia nuclear magnética de abdomen la cual mostró una lesión hepática lobulada de contornos bien definidos, hiperintensa, localizada en el segmento VI, con restricción de la difusión y realce periférico nodular progresivo, sin zonas de "lavado" relacionada con presentación típica de hemangioma; además, se evidenciaron alteraciones múltiples en el patrón de perfusión hepático, en relación con estructuras vasculares irregulares que comprometían principalmente los segmentos
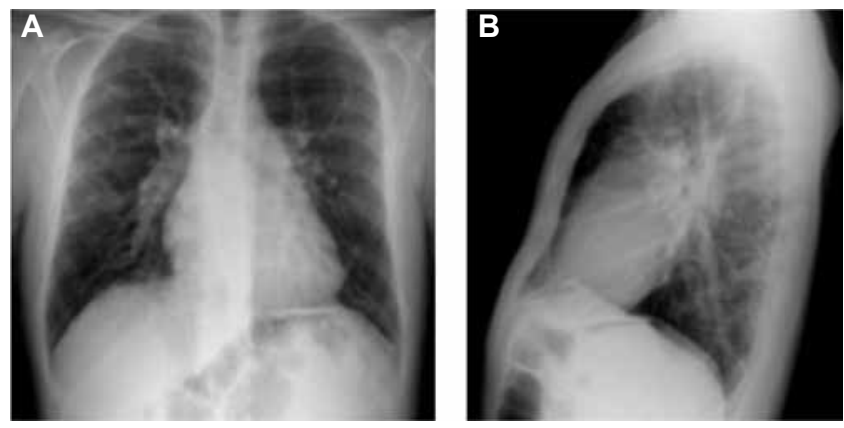

Figura 2. Paneles A y B. Radiografía de tórax en la que se observa dilatación de los segmentos principales de las arterias pulmonares. 
superiores, sugestivo de pequeños cortocircuitos arterio-venosos, secundarios a malformaciones vasculares (figura 5).

\section{Discusión}

Se conocen cerca de 600 mutaciones asociadas con esta enfermedad, dentro de las que se destacan las ateraciones genéticas relacionadas con deficiencia o deterioro de la función de las moléculas de endoglina o activina tipo receptor kinasa 1 (ALK-1) (2). Ambos componentes se expresan en las células endoteliales vasculares y su defecto conduce a ARN mensajero y proteínas celulares inestables. La falta de estas proteínas en el 50\% conduce a una función anormal en la mitad de las células vasculares.
Estos genes y proteínas están implicados en la señalización de una superfamilia de factores de crecimiento transformante beta (TGF- $\beta$ ) (3). Este grupo de factores de crecimiento interviene en el desarrollo y la diferenciación celular, a través de señales de receptores y complejos transmembrana, que se traslocan dentro del núcleo, donde modulan la expresión de genes diana por medio de la unión directa al ADN.

La ALK-1 es un receptor TGF- $\beta$ tipo I, que se relaciona durante la señalización con la endoglina y modifica las señales durante el proceso de desarrollo vascular generando un fenotipo celular que se asocia con telangiectasias, malformaciones y aneurismas vasculares (4). Esta situación, en conjunto con los defectos de la proteína morfogénica ósea (BMPR2),
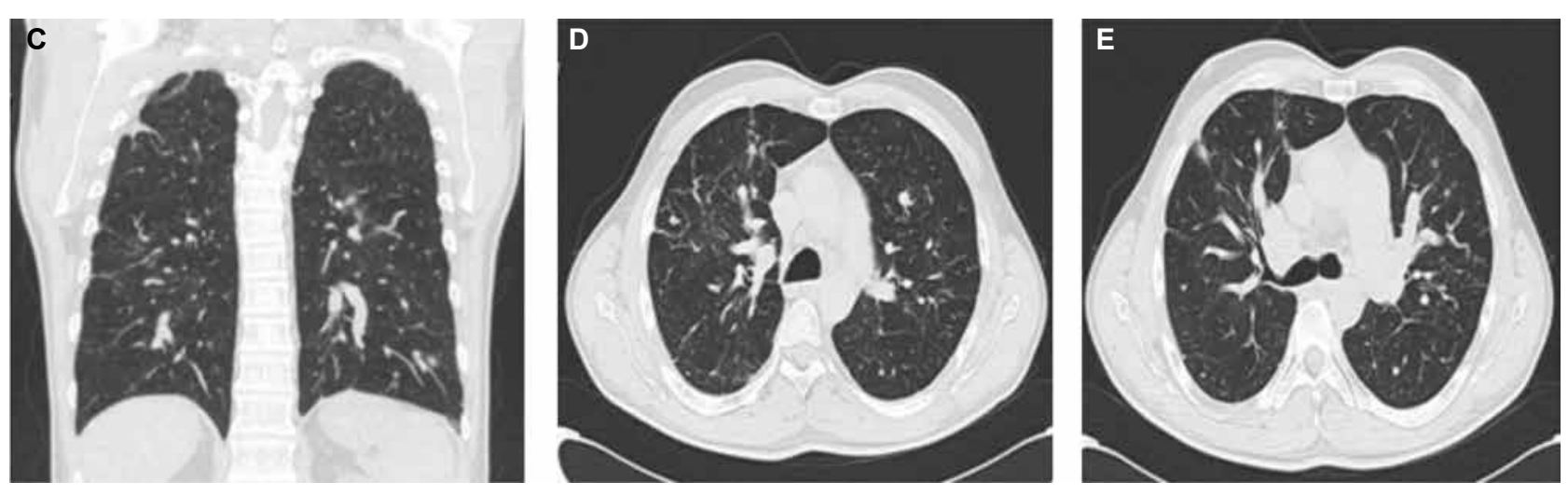

Figura 3. Paneles C, D y E. Tomografía de tórax que muestra imágenes de aspecto nodular ovaladas con diámetros entre 3 y $5 \mathrm{~mm}$.

A

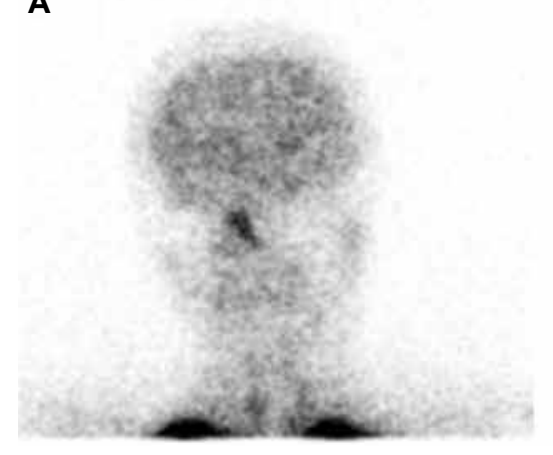

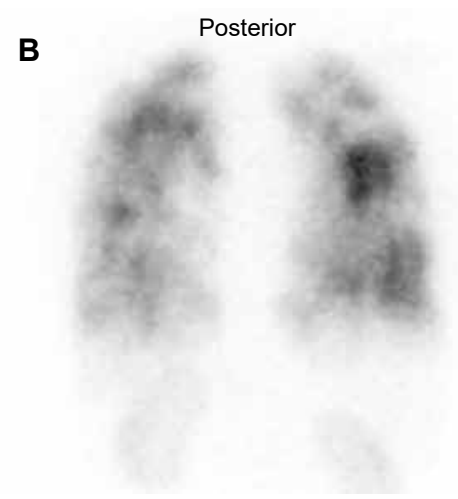

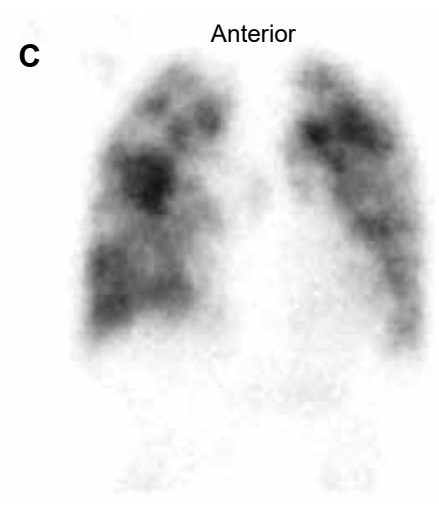

Figura 4. Paneles A, B y C. Gammagrafía en la que observan macroagregados de albúmina en el cerebro y distribución irregular en áreas del parénquima pulmonar. 
se han encontrado de forma similar en los pacientes con hipertensión pulmonar primaria (5), lo cual genera un fenotipo clínico indistinguible de la hipertensión causada por las malformaciones arteriovenosas pulmonares y el estado de circulación hiperdinámico presente en esta entidad (6).

Algunas manifestaciones clínicas ocurren desde la infancia, aproximadamente en el $70 \%$ de los casos, y otras se hacen presentes en la edad adulta. La epistaxis es el primer síntoma de la enfermedad, ya que las telangiectasias son el componente inicial. El compromiso gastrointestinal, cerebral, pulmonar $\mathrm{y}$ hepático secundario a las malformaciones arteriovenosas se da de manera progresiva con la edad haciéndose más evidente durante la pubertad y en la edad adulta $(7,8)$.

Ahora se centrará la atención en el compromiso pulmonar de la enfermedad, dado que este no es despreciable. Las malformaciones arteriovenosas pulmonares se encuentran en el $45 \%$ de los casos. Estas se caracterizan por anormalidades de los vasos que tienen paredes delgadas y sustituyen a los capilares normales entre la arteria pulmonar y la circulación venosa, generando estructuras parecidas a sacos bulosos, que permiten una comunicación directa entre las circulaciones pulmonar y sistémica (9), a través de los cuales el flujo sanguíneo pasa formando un cortocircuito de derecha a izquierda; la sangre que atraviesa esta zona queda sin ser oxigenada y conduce a hipoxemia (10). La hipertension pulmonar es otra complicación que sucede en el $10 \%$ de los pacientes y puede instaurarse por mecanismos como el alto gasto cardíaco debido al cortocircuito de derecha a izquierda, el cual genera una presión alta de oclusión en la arteria pulmonar con resistencia vascular pulmonar normal. La presión y el flujo pulmonar elevados predisponen a la ampliación de las malformaciones arteriovenosas (11).

Los pacientes pueden tener hipertensión portopulmonar debido a la hipertensión portal causada por las malformaciones hepáticas. En estos casos la presión de enclavamiento de la arteria pulmonar es normal, con resistencia vascular pulmonar incrementada (12).

Debido a los múltiples orígenes de esta condición, el estudio hemodinámico es una herramienta útil en el diagnóstico diferencial de la misma (13).
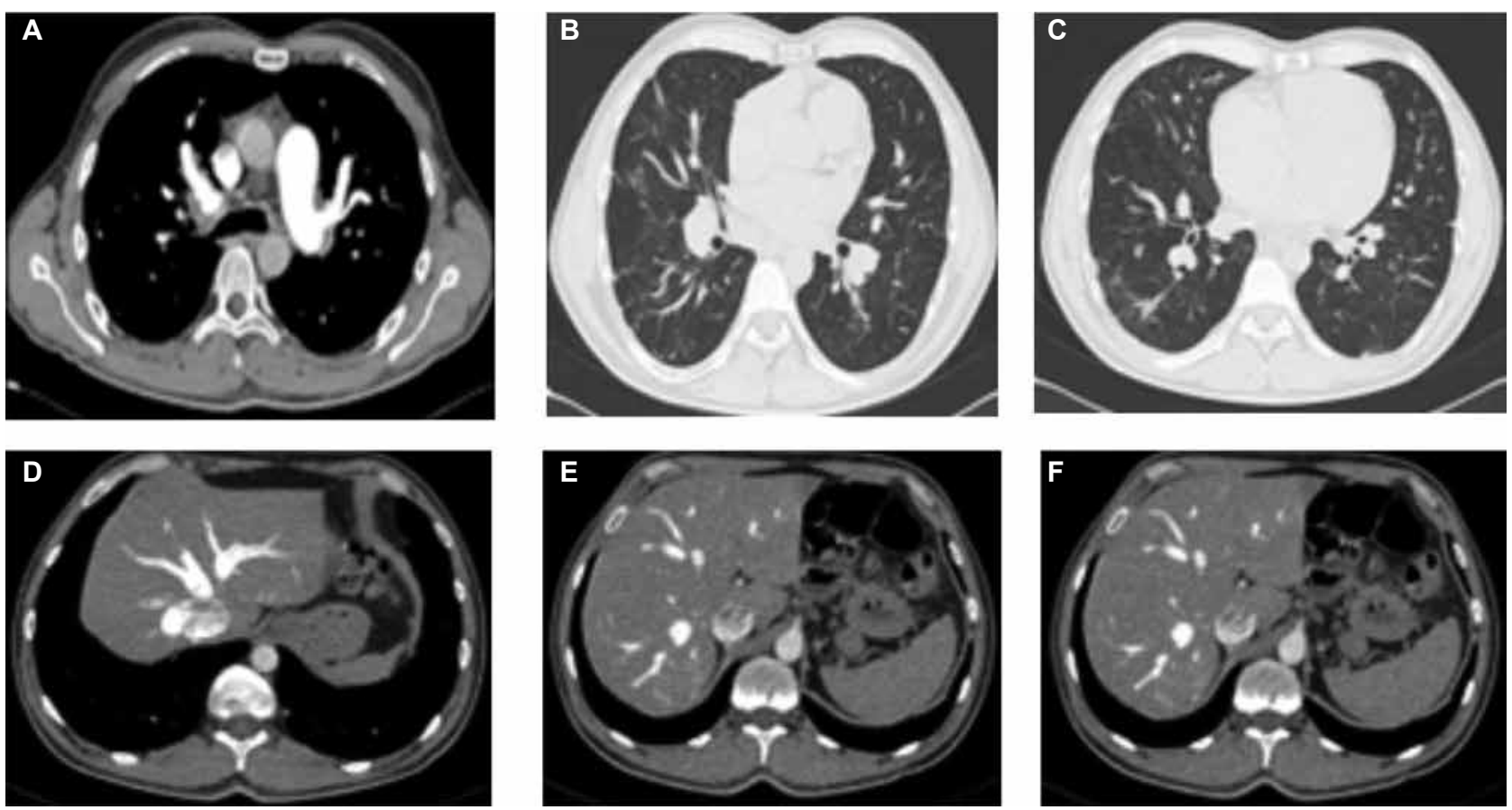

Figura 5. Paneles A, B y C. Resonancia nuclear magnética de tórax con contraste intravenoso, donde se ponen en manifiesto las malformaciones arteriovenosas pulmonares y hepáticas. Paneles D, E y F; se observan malformaciones vasculares hepáticas. 
Tabla 1. Criterios diagnósticos de Curaçao para la telangiectasia hemorrágica hereditaria.

Diagnóstico basado en los criterios del Consenso internacional (criterios diagnósticos Curaçao)
- $\quad$ Epistaxis o hemorragia nasal espontánea y recurrente
- Telangiectasias en múltiples sitios característicos
(labios, cavidad oral, dedos, nariz).
- Lesiones viscerales
$\quad$ Telangiectasias en el tracto gastrointestinal (con o sin sangrado)
$\quad$ Malformaciones arteriovenosas pulmonares, hepáticas, cerebrales, espinales.
- Antecedentes familiares:
Familiar en primer grado con diagnóstico de THH
Los criterios clasifican la enfermedad en:
1. "Definitiva" si se cumplen tres criterios.
2. "Posible o presunta" si se cumplen dos criterios.
3. "Poco probable" si se cumplen menos de dos criterios.

Por otro lado, la coexistencia de hipertension pulmonar y THH, pueden estar relacionadas con defectos en endoglina, ALK-1 o BMPR2 dado que sus alteraciones se presentan tanto en una condicion como en la otra de manera indistinguible (14).

Los síntomas asociados a estas condiciones pueden incluir: disnea, uno de los síntomas principales y que se manifiesta cuando las malformaciones son de gran tamaño, bilaterales o difusas (15): hemoptisis, que va desde leve hasta severa, y empeora en condiciones que aumenten el volumen sanguíneo circulante o el gasto cardíaco, como el embarazo (16); platipnea (aumento de la disnea inducida por la posición vertical y aliviada por el decúbito) y ortodesoxia (una disminución de la saturación de oxihemoglobina al levantarse de la posición supina a la posición vertical). Se cree que la platipnea es el resultado del aumento del flujo sanguíneo a través de las malformaciones arteriovenosas en las porciones inferiores de los pulmones al asumir la posición vertical. Con el fin de realizar un diagnóstico adecuado de esta patología se han descrito los criterios de Curaçao (17) (tabla 1).

Por último se hará referencia a los hallazgos imagenológicos dado que de esta forma se realizó el diagnóstico de la paciente del caso. La mayoría se presenta con radiografías de tórax anormales atribuibles a malformaciones arteriovenosas, cuyas características principales son masas redondas ovaladas delimitadas, de densidad uniforme y ocasionalmente lobuladas, como las que se reportan en el caso. Por lo general, miden de 1 a $5 \mathrm{~cm}$ de diámetro, pero pueden llegar hasta los 9 a $10 \mathrm{~cm}$ de diámetro, en cantidad de dos a ocho lesiones, algunas acompañadas de vaso nutricio (18).

En cuanto a la tomografía computarizada es más sensible que la radiografía de tórax para detectar estas alteraciones, ya que puede identificarlas casi en su totalidad, incluso en aquellos que no presentan alteraciones en las radiografías de tórax convencionales (19). A veces se requiere angiografía pulmonar e imágenes con realce a través de medio de contraste para el diagnóstico definitivo, como ocurrió en este caso. La gammagrafía con macroagregados de albúmina en el cerebro y en los riñones indica presencia de cortocircuito de derecha a izquierda (20-21).

Así, mientras que una radiografía de tórax completamente normal hace que el diagnóstico sea menos probable, no se debe excluir la evaluación con tomografía computarizada o angiografía, particularmente en un paciente con otras características comunes a la THH.

\section{Bibliografía}

1. Bayrak-Toydemir P, McDonald J, Akarsu N, Toydemir RM, Calderon F, Tuncali T. A fourth locus for hereditary hemorrhagic telangiectasia maps to chromosome 7. Am J Med Genet A. 2006; 140:2155. 
2. Harrison RE, Flanagan JA, Sankelo M, Abdalla SA, Rowell $\mathrm{J}$, Machado RD. Molecular and functional analysis identifies ALK-1 as the predominant cause of pulmonary hypertension related to hereditary haemorrhagic telangiectasia. J Med Genet. $2003 ; 40: 865$.

3. Trembath RC, Thomson JR, Machado RD, Morgan NV, Atkinson C, Winship I. Clinical and molecular genetic features of pulmonary hypertension in patients with hereditary hemorrhagic telangiectasia. N Engl J Med. 2001;345:325.

4. Bertolino P, Deckers M, Lebrin F, Ten Dijke P. Transforming growth factor-beta signal transduction in angiogenesis and vascular disorders. Chest. 2005; 128:585S.

5. Kajdaniuk D, Marek B, Borgiel-Marek H, Kos-Kudla B. Transforming growth factor $\beta 1$ (TGF $\beta 1$ ) in physiology and pathology. Endokrynol Pol. 2013;64(5):384-96.

6. Abdalla SA, Gallione CJ, Barst RJ, Horn EM, Knowles JA, Marchuk DA. Primary pulmonary hypertension in families with hereditary haemorrhagic telangiectasia. Eur Respir J. 2004;23:373.

7. Fuchizaki U, Miyamori H, Kitagawa S, Kaneko S, Kobayashi $\mathrm{K}$. Hereditary haemorrhagic telangiectasia (Rendu-Osler-Weber disease). Lancet 2003; 362:1490.

8. Govani FS, Shovlin CL. Hereditary haemorrhagic telangiectasia: a clinical and scientific review. Eur J Hum Genet. 2009; 17:860.

9. Van Gent MW, Post MC, Snijder RJ, Westermann CJ, Plokker HW, Mager JJ. Real prevalence of pulmonary right-to-left shunt according to genotype in patients with hereditary hemorrhagic telangiectasia: a transthoracic contrast echocardiography study. Chest. 2010;138:833.

10. Shovlin C, Bamford K, Wray D. Post-NICE 2008: Antibiotic prophylaxis prior to dental procedures for patients with pulmonary arteriovenous malformations (PAVMs) and hereditary haemorrhagic telangiectasia. Br Dent J. 2008;205:531.

11. Porres-Aguilar M, Altamirano JT, Torre-Delgadillo A, Charlton MR, Duarte-Rojo A. Portopulmonary hypertension and hepatopulmonary syndrome: a clinician-oriented overview. Eur Respir Rev. 2012;21:223-33.

12. Krowka MJ. Hepatopulmonary syndrome and portopulmonary hypertension. Curr Treat Options Cardiovasc Med. 2002;4:267.

13. Faughnan ME, Palda VA, Garcia-Tsao G, Geisthoff UW, McDonald J, Proctor DD. International guidelines for the diagnosis and management of hereditary haemorrhagic telangiectasia. J Med Genet. 2011;48:73.

14. Shovlin CL. Hereditary haemorrhagic telangiectasia: pathophysiology, diagnosis and treatment. Blood Rev. 2010;24:203.

15. Plauchu H, de Chadarévian JP, Bideau A, Robert JM. Agerelated clinical profile of hereditary hemorrhagic telangiectasia in an epidemiologically recruited population. Am J Med Genet. 1989;32:291.

16. Shovlin CL, Sodhi V, McCarthy A, Lasjaunias P, Jackson JE, Sheppard MN. Estimates of maternal risks of pregnancy for women with hereditary haemorrhagic telangiectasia (OslerWeber-Rendu syndrome): suggested approach for obstetric services. BJOG. 2008;115:1108.

17. Westermann CJ, Rosina AF, De Vries V, de Coteau PA. The prevalence and manifestations of hereditary hemorrhagic telangiectasia in the Afro-Caribbean population of the Netherlands Antilles: a family screening. Am J Med Genet. 2003;116A:324.

18. Elliott CG, Barst RJ, Seeger W, Porres-Aguilar M, Brown LM, Zamanian RT. Worldwide physician education and training in pulmonary hypertension: pulmonary vascular disease: the global perspective. Chest. 2010;137:85S-94S.

19. Herzer K, Post F, Canbay A, Gerken G. Pulmonary affection in advanced liver disease - hepatopulmonary syndrome and portopulmonary hypertension. Med Klin (Munich). 2010;105(12):916-23.

20. Seto H, Futatsuya R, Kamei T, Kakishita M, Seki M, Yamamoto K, Tanaka H. Pulmonary arteriovenous malformation: radionuclide detection and quantification of right-to-left shunting. Radiat Med 1985;3(1):33-7.

21. Ones T1, Dede F, Erdim R, Erdil TY, Inanir S, Yuksel M, Turoglu HT. Quantitative shunt imaging in the evaluation of therapeutic surgery in a patient with pulmonary arteriovenous malformation. Ann Surg Thorac. 2008;86(2):649-51. 